\title{
Monte Carlo Simulation for the Morphology and Kinetics of Spherulites and Shish-Kebabs in Isothermal Polymer Crystallization
}

\author{
Chunlei Ruan, ${ }^{1,2}$ Chuntai Liu, ${ }^{3}$ and Guoqiang Zheng ${ }^{2}$ \\ ${ }^{1}$ School of Mathematics and Statistics, Henan University of Science and Technology, Luoyang 471023, China \\ ${ }^{2}$ College of Materials Science and Engineering, Zhengzhou University, Zhengzhou 450002, China \\ ${ }^{3}$ National Engineering Research Center for Advanced Polymer Processing Technology, Zhengzhou 450001, China \\ Correspondence should be addressed to Chunlei Ruan; ruanchunlei622@mail.nwpu.edu.cn
}

Received 7 April 2015; Revised 19 June 2015; Accepted 28 September 2015

Academic Editor: Carla Roque

Copyright ( 2015 Chunlei Ruan et al. This is an open access article distributed under the Creative Commons Attribution License, which permits unrestricted use, distribution, and reproduction in any medium, provided the original work is properly cited.

\begin{abstract}
Monte Carlo method is used to capture the evolution of spherulites and shish-kebabs and to predict the crystallization kinetics in isothermal polymer crystallization. Effects of nucleation density and growth rate of spherulites, nucleation density, and length growth rate of shish-kebabs, respectively, on crystallization are investigated. Results show that nucleation densities of both spherulites and shish-kebabs strongly affect crystallization rate as well as morphology. An increase in nucleation density of either spherulites or shish-kebabs leads to a quicker crystallization rate and a smaller average spherulite size. It is also shown that nucleation density of shish-kebabs has a stronger impact on crystallization rate. Growth rate of spherulites and length growth rate of shish-kebabs also have significant effect on crystallization rate and morphology. An increase in growth rate of spherulites or length growth rate of shish-kebabs also speeds up the crystallization rate; additionally, a decrease in growth rate of spherulites or an increase in length growth rate of shish-kebabs results in a more highly anisotropic shish-kebab structure and a smaller average size of spherulites. Results also show that the effect of growth rate of spherulites is more important than the effect of length growth rate of shish-kebabs on crystallization.
\end{abstract}

\section{Introduction}

This study is motivated by an interest in the crystallization of semicrystalline polymers during the manufactural processing. Usually, these materials are injected as viscous/viscoelastic melt into a mold that gives it the desired final shape. The resulting product is then cooled to make a solid composite with a typical crystalline structure that is a key feature of the finished product since it affects mechanics, thermomechanics of the materials [1]. Therefore it is of great importance to predict the crystal morphology and crystallinity formed during the manufactural processing.

Experimental results show that the crystalline structure in final injection product takes on a typical "skin-core-skin" structure [1], that is, a shish-kebab skin with highly orientation and a spherulitic core with essentially no preferred orientation. These two types of crystals are formed depending on the mechanics and mechanical history. For example, in the center of a product, because of the absence/lack of shear, the random polymer chain leads to lamellar, chain folded crystals and finally the spherulites; while near the walls, due to the highly shear or strain, the extended polymer chain leads to extended chain crystals and finally shishkebab structures. This is also known since spherulites are related to quiescent crystallization, while shish-kebabs are related to flow-induced crystallization $[1,2]$. Hence, to predict crystal morphology accurately in the manufactural processing, it is necessary to combine spherulites with shishkebabs.

There have been many developments in the modeling of flow-induced crystallization. Researchers proposed different models to describe the effect of flow on crystallization kinetics based on Nakamura approach or Avrami-Kolmogoroff approach [2]. Models based on Nakamura approach do not 
take morphology development into account but predict the crystallization kinetics well. For example, a multiplying factor function of stress [3], shear rate [4], strain [4], orientation [5], and so forth was applied to modify the crystallization kinetic constant in the original Nakamura model to include the effect of flow on crystallization. On the other hand, models based on Avrami-Kolmogoroff approach do consider the crystallization phenomenon as a consequence of nucleation and growth. According to many researches, flow has minor effect on crystal growth [2]. Therefore, many efforts were made to find the driving force for flow-induced nucleation. For example, parameters including shear rate [4], recoverable strain tensor [6], first normal stress difference [7], and free energy [8] were used to reflect the effect of flow on crystallization. However, we will mention that AvramiKolmogoroff model can not provide information on the final crystalline structure when spherulites and shish-kebabs coexist. The parameters in Avrami-Kolmogoroff model, for example, $C_{m}$ (shape factor), $m$ (dimensionality of growth), are often found by data fitting [8] which lose the clear information of crystalline structure.

To predict in detail the crystalline structure forming, researchers proposed different methods. For example, Swaminarayan and Charbon [9] presented a normal front-tracking method and a radial front-tracking method to describe the evolution of a limited set of points on crystallization front; Capasso [10], Micheletti and Burger [11], and Ketdee and Anantawaraskul [12] developed a stochastic birth-and-growth process to simulate the isother$\mathrm{mal} /$ nonisothermal crystallization in polymers; Raabe [13], Raabe and Godara [14], and Lin et al. [15] proposed a cellular automaton model to predict the spherulite growth and spatial distribution in polymers; Ruan et al. [16-18] presented a "pixel coloring" method to simulate the crystal morphology forming and kinetics calculation in short-fiber reinforced systems and pure polymer systems. However, these works are only concentrated on quiescent crystallization, in which the object of crystalline structure is spherulite.

So far, a few works have been done to deal with the morphology evolution of spherulites and shish-kebabs. Zuidema et al. [6] used Eder model and Schneider rate equations to describe the evolution of spherulites and shish-kebabs, respectively, and visualized the development of crystalline structure by pixel. Their work has taken a huge step in the modeling and simulation of crystalline structure forming including flow-induced crystallization. However, they did not show the detailed implement of capturing crystal fronts; besides, the microstructure (total volume, surface, and length of shish-kebabs) predicted by Schneider rate equations was based on fiction where overlapping was allowed [8].

The objective of this paper is to present a Monte Carlo simulation to capture the evolution of crystalline structure (spherulites and shish-kebabs), to predict the final structure spatial distribution, and to calculate the crystallization rate in isothermal polymer crystallization. We will mention that this isothermal crystallization is a simplification in a real manufactural processing but is the case that happens in some experiments [2].

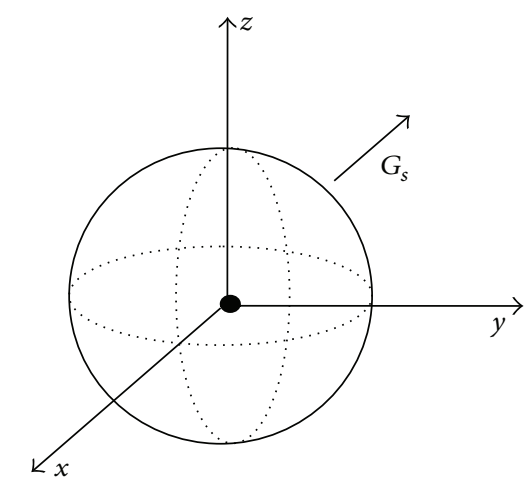

Figure 1: Schematic growth of single spherulite.

\section{Theory and Modeling}

Avrami model is the most widely used one describing the macroscopic evolution of crystallinity with time for various modes of nucleation and growth in isothermal polymer system. It can be expressed as follows [19-21]:

$$
\alpha=1-\exp \left(-\alpha_{f}\right)
$$

where $\alpha$ is the relative crystallinity which takes into account impingement due to space filling effect; $\alpha_{f}$ is a fictive volume fraction of "phantom crystals" at time $t$ in the case overlapping is allowed.

We assume the polymer is undergoing the shear and it is sufficient to allow the polymer to generate the shish-kebabs. Besides, the crystallization occurs at a constant relative low temperature and the spherulites will appear. Therefore, in this case, the crystalline structures are the spherulite and shishkebab.

These two kinds of structures contribute to the fictive volume fraction and can be combined by simply adding up

$$
\alpha_{f}=\alpha_{s}+\alpha_{\mathrm{s}-\mathrm{k}}
$$

here, $\alpha_{s}$ is the fictive volume fraction of spherulites and $\alpha_{s-\mathrm{k}}$ is the fictive volume fraction of shish-kebabs.

Under the isothermal crystallization, the nucleation of spherulites is assumed to take place at $t=0$ with the nucleation density $N_{s}$. According to many researches, flow has minor effect on the growth rate of spherulites. Hence, it also follows the Hoffman-Lauritzen theory [8] from which we know that the growth rate of spherulites $G_{s}$ under the isothermal case is constant. Therefore, the fictive volume fraction of spherulites can be written as

$$
\alpha_{s}=\frac{4 \pi}{3} N_{s}\left(G_{s} t\right)^{3}
$$

with $N_{s}$ being the nucleation density of spherulites and $G_{s}$ being the growth rate of spherulites. Figure 1 gives the schematic growth of single spherulite.

On the other hand, we assume shish-kebabs growth like cylinders. This is also the way that Zuidema et al. [6] did. Figure 2 gives the schematic growth of single shish-kebab. We assume that at $t=0$ there is a relative high shear rate (larger than the critical shear rate) to generate the shish-kebabs with 


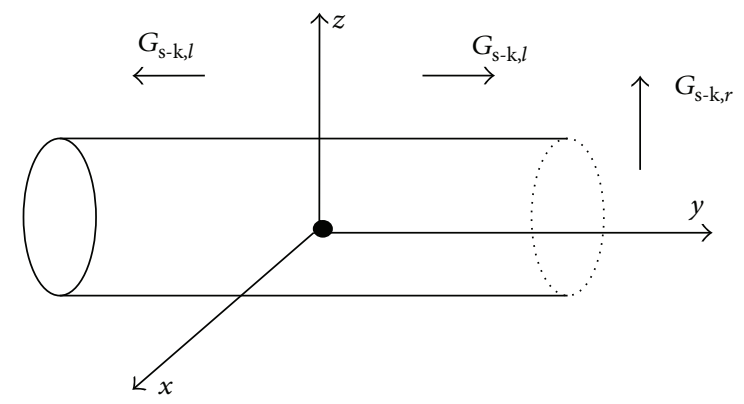

FIGURE 2: Schematic growth of single shish-kebab.

the nucleation density $N_{s-\mathrm{k}}$. After the nucleation, the shear rate is keeping under the critical shear rate which cannot contribute to nucleation but does affect the growth of shishkebabs. According to Zuidema et al. [6], the radius growth rate of shish-kebabs $G_{s-k, r}$ is related to temperature. Therefore, under the isothermal case, this parameter is constant. Besides, the length growth rate of shish-kebabs $G_{s-\mathrm{k}, l}$ is related to flow [6]. With the help of Schneider rate equation [6] and the hypothesis of constant shear rate, we see that the length growth rate of shish-kebabs $G_{s-k, l}$ treated as constant is reasonable. Therefore, the fictive volume fraction of shishkebabs can be computed as

$$
\alpha_{s-\mathrm{k}}=\pi N_{\mathrm{s}-\mathrm{k}}\left(G_{s-\mathrm{k}, r} t\right)^{2}\left(2 G_{\mathrm{s}-\mathrm{k}, \mathrm{l}} t\right),
$$

where $N_{s-k}$ is the nucleation density of shish-kebabs; $G_{s-k, r}$, $G_{s-k, l}$ are the radius and length growth rate of shish-kebabs, respectively.

It can be seen from (1)-(4) that six variables can completely define an isothermal polymer crystallization (quiescent and flow-induced crystallization). These are $N_{s}, G_{s}, N_{s-k}$, $G_{s-k, r}, G_{s-k, l}$, and $t$. Therefore, a parametric study is used here to explore the effects of each variable on the crystal morphology evolution as well as crystallization kinetics.

Monte Carlo method also known as stochastic simulation is often adopted as a compute mathematical problem based on the statistical value of plenty of random points. The basic idea of this method is expressed as follows: firstly, creating a random probability model or random process according to the given problem and letting the parameter be equal to the solution of the problem; secondly, calculating the statistical characteristics of parameters by doing observation or sample experiments for the created model or process; finally, computing the approximate value of the solution.

In this problem, we assume the crystalline phase and amorphous phase distribute uniformly in space; therefore, the relative crystallinity $\alpha$ can be treated as degree of space filling which can be expressed as the following form:

$$
\alpha=\frac{v_{c}}{v_{\text {tot }}},
$$

where $v_{c}$ is a time-dependent value which means the volume of the crystallized grains at time $t$ and $v_{\text {tot }}$ is the total volume. In order to obtain $\alpha$, we build up the following process:

(1) Divide the computational zone as a large array of equally sized cubes with each volume $v$ and total number $N_{\text {tot }}$.
(2) Produce the random nuclei center with a nucleation density of spherulites $N_{s}$ and a nucleation density of shish-kebabs $N_{\mathrm{s}-\mathrm{k}}$ at $t=0$. Assume that each nucleation occupies one unit cell.

(3) For each time step $\left(t_{j}, t_{j+1}\right)$, calculate the radius of spherulites as $R_{s}=G_{s} \times t_{j+1}$, the radius of shishkebabs as $R_{s-\mathrm{k}}=G_{s-\mathrm{k}, r} \times t_{j+1}$, and the length of shish-kebabs $L_{s-\mathrm{k}}=2 G_{\mathrm{s}-\mathrm{k}, l} \times t_{j+1}$. Produce a large number of random points restricted to the center of cells. For each random point, if it falls within the range of radius of one sphere, its belonging cell is changed to a crystalline cell and considered to be occupied by that spherulite; if it falls within the range of radius and length of one cylinder, its belonging cell is changed to a crystalline cell and considered to be occupied by that shish-kebab. In the case that if a random point falls within the range of radius or length of several crystals, its belonging cell is assumed to be occupied by the crystals having the minimal time to reach that point.

(4) Calculate $\alpha$ by $\alpha=v_{c} / v_{\text {tot }}=\left(N_{c} v\right) /\left(N_{\text {tot }} v\right)=$ $N_{c} / N_{\text {tot }}$ with $N_{c}$ the number of cells that have been transformed.

(5) Go to third step until $t=t_{\text {end }}$ or $\alpha=1$.

The above process, especially step three, presents a random process, which allows us to give an approximation of $\alpha$ by Monte Carlo method.

\section{Results and Discussion}

The crystallization kinetics and morphology are studied by Monte Carlo method in a small spatial region, $[0,1] \mathrm{mm} \times$ $[0,1] \mathrm{mm} \times[0,1] \mathrm{mm}$ in particular. Unless otherwise stated, we set the parameters as $N_{\text {tot }}=10^{7}, N_{s}=N_{\mathrm{s}-\mathrm{k}}=10^{3} / \mathrm{mm}^{3}$, $G_{s}=G_{s-\mathrm{k}, r}=1 \mu \mathrm{m} / \mathrm{s}$, and $G_{s-\mathrm{k}, l}=10 \mu \mathrm{m} / \mathrm{s}$. It is worth noting that the radius growth of shish-kebabs is in a similar manner to spherulitic growth according to many experiments [1]. Hence, we set $G_{s}=G_{s-k, r}$ in this study. Therefore, we only explore the effects of $N_{s}, G_{s}, N_{s-k}$, and $G_{s-k, l}$ in the following investigation. This algorithm is written in $\mathrm{C}++$ langue and runs on Personal Computer.

3.1. Validity of the Simulation. Figure 3 shows the comparison of relative crystallinity of Monte Carlo result with Avrami model. Evidently, the Monte Carlo result agrees well with Avrami model. This confirms the validity of our algorithm.

Using the results of Monte Carlo method, we can visualize the development of spherulites and shish-kebabs. These are shown in Figures 4 and 5, where spherulite is represented by a sphere while shish-kebab is treated as a cylinder. Crystals grow individually without impingement (see Figures 4(a) and 5(a)) at the early time; however, as the time increases, several impingement faces occur (see Figures 4(b), 4(c), 5(b), and 5(c)) until reaching the final morphology (see Figures 4(d) and 5(d)). The final morphology shows a qualitative agreement with the experimental results of Zheng et al. [22], 
TABLE 1: Volume fraction and average length-to-diameter ratio of shish-kebabs.

\begin{tabular}{lccc}
\hline Case & $N_{s}=10^{2} / \mathrm{mm}^{3}$ & $N_{s}=10^{3} / \mathrm{mm}^{3}$ & $N_{s}=10^{4} / \mathrm{mm}^{3}$ \\
\hline Volume fraction of shish-kebabs & $99.2 \%$ & $92.2 \%$ & $54.4 \%$ \\
Average length-to-diameter ratio of shish-kebabs & 8.3 & 7.93 & 7.90 \\
\hline
\end{tabular}

TABLE 2: Volume fraction and average length-to-diameter ratio of shish-kebabs.

\begin{tabular}{lccc}
\hline Case & $N_{s-\mathrm{k}}=10^{2} / \mathrm{mm}^{3}$ & $N_{\mathrm{s}-\mathrm{k}}=10^{3} / \mathrm{mm}^{3}$ & $N_{\mathrm{s}-\mathrm{k}}=10^{4} / \mathrm{mm}^{3}$ \\
\hline Volume fraction of shish-kebabs & $51.8 \%$ & $92.2 \%$ & $99.2 \%$ \\
Average length-to-diameter ratio of shish-kebabs & 7.17 & 7.93 & 8.09 \\
\hline
\end{tabular}

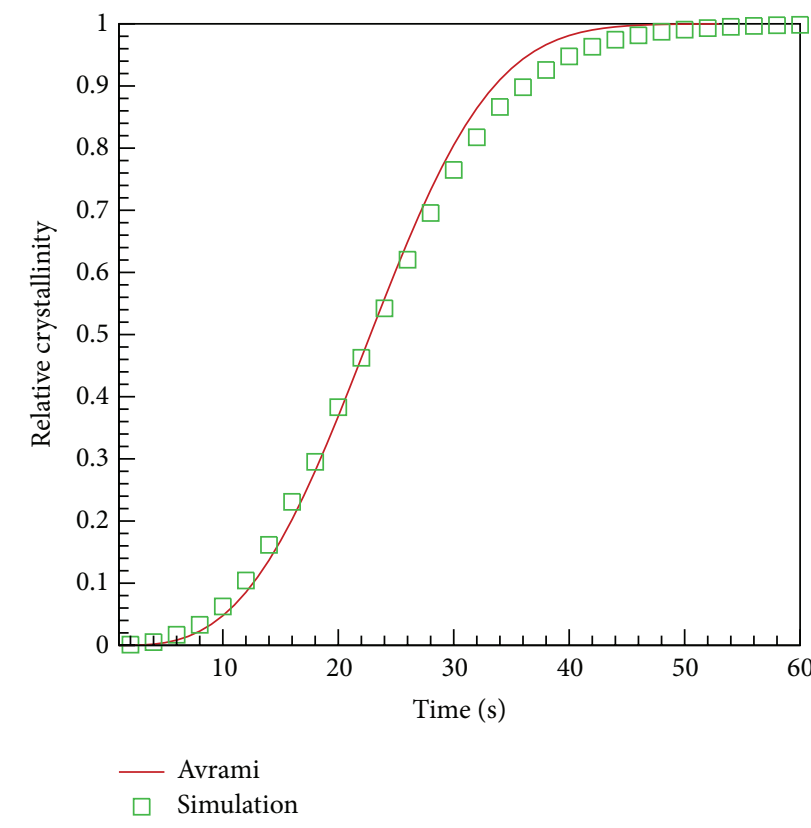

Figure 3: Comparison of Monte Carlo result with analytic Avrami model.

Zhou et al. [23], and the numerical results of Zuidema et al. [6].

3.2. Effects of Nucleation Density of Spherulites $N_{s}$. Figure 6 shows the effects of nucleation density of spherulites $N_{s}$ on crystallization kinetics and morphology. We change the nucleation density of spherulites $N_{s}$ varying from $10^{2} / \mathrm{mm}^{3}$ to $10^{3} / \mathrm{mm}^{3}$ and then to $10^{4} / \mathrm{mm}^{3}$ while holding other parameters fixed to allow comparison. It is noticeable that the time to reach complete crystallization decreases with increasing nucleation density of spherulites. This is consistent with the theoretical analysis of Avrami model which is presented in (1)-(4) in this study. Morphology for similar relative crystallinity $(\alpha \approx 0.5)$ with different nucleation density of spherulites is also shown in Figure 6. As we can see, due to the increasing of nucleation density of spherulites, crystals are more likely to impinge.

Figure 7 gives the radius distribution of spherulites in the final morphology. The average radius of single spherulite is calculated by $\bar{R}=\sqrt[3]{3 v_{s} /(4 \pi)}$ where $v_{s}$ is the volume of single spherulite and can be obtained by the number of cells belonging to this spherulite and the cell volume. It can be found in Figure 7 that the mean radius decreases with the increase of nucleation density of spherulites. Volume fraction and the average length-to-diameter ratio of shishkebabs in the final morphology are listed in Table 1. It should be mentioned that the average length-to-diameter ratio of shish-kebabs is difficult to calculate. We here assume the radius of shish-kebabs is equal to the average radius of spherulites. This is reasonable since this simulation is carried out under the assumption that the radius growth of shish-kebabs equals spherulitic growth rate. From Table 1, we see that the increasing of nucleation density of spherulites decreases the volume fraction of shish-kebabs. Besides, its effect on the average length-to-diameter ratio of shish-kebabs is minor.

In the real polymer processing, the nucleation density of spherulites $N_{s}$ is directly determined by temperature. Therefore, one can change the temperature to adjust the nucleation density of spherulites, thereby to obtain the desired crystal size and crystallization rate.

3.3. Effects of Nucleation Density of Shish-Kebabs $N_{\mathrm{s}-\mathrm{k}}$. Figure 8 shows the effects of nucleation density of shishkebabs $N_{s-k}$ on crystallization kinetics and morphology. We change nucleation density of shish-kebabs $N_{\mathrm{s}-\mathrm{k}}$ from $10^{2} / \mathrm{mm}^{3}$ to $10^{3} / \mathrm{mm}^{3}$ and then to $10^{4} / \mathrm{mm}^{3}$. It is notable that increasing the nucleation density of shish-kebabs leads to an acceleration of the crystallization. By comparing with Figure 6, it is clear that the effect of nucleation density of shish-kebabs on crystallization kinetics is more important than that nucleation density of spherulites. Since the shishkebab structure is highly anisotropic, its length growth rate $G_{\mathrm{s}-\mathrm{k}, l}$ is much larger than its radius growth rate $G_{\mathrm{s}-\mathrm{k}, r}$ which is assumed to be equal to the growth rate of spherulites $G_{s}$. Therefore, the volume of shish-kebabs increases faster than the spherulites which lead to a stronger effect on crystallization kinetics.

Radius distribution of spherulites in the final morphology is descripted in Figure 9. It is obvious that, in the case of lower nucleation density of shish-kebabs, the average spherulite size is larger. Table 2 shows the volume fraction and the average length-to-diameter ratio of shish-kebabs in the final morphology. It is evident that volume fraction of shishkebabs increases with the increase of nucleation density of 


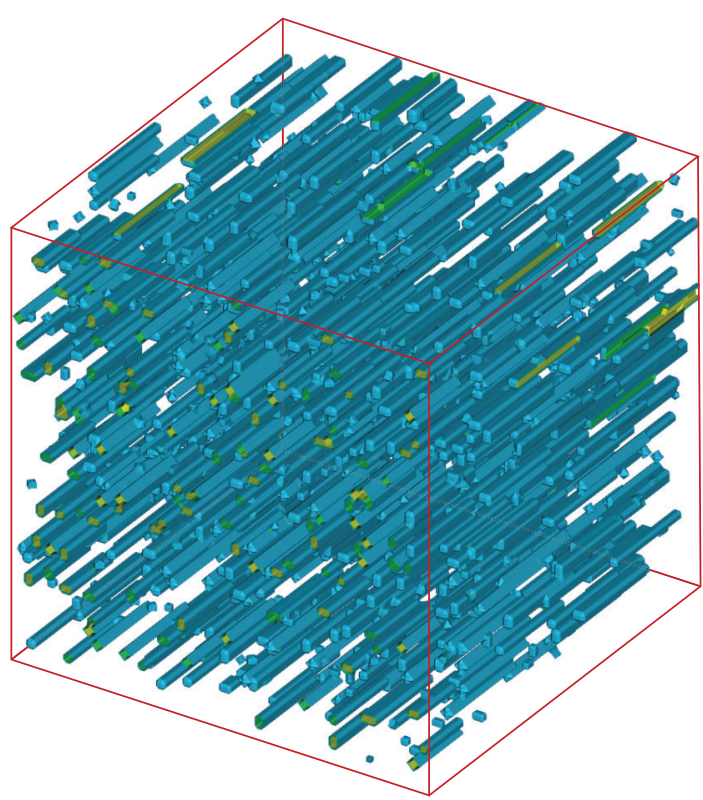

(a)

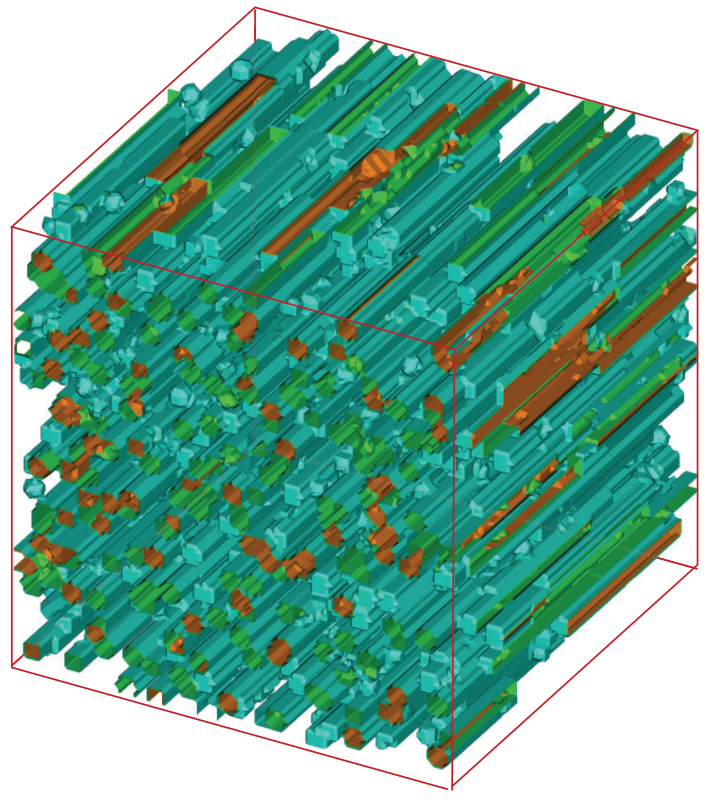

(c)

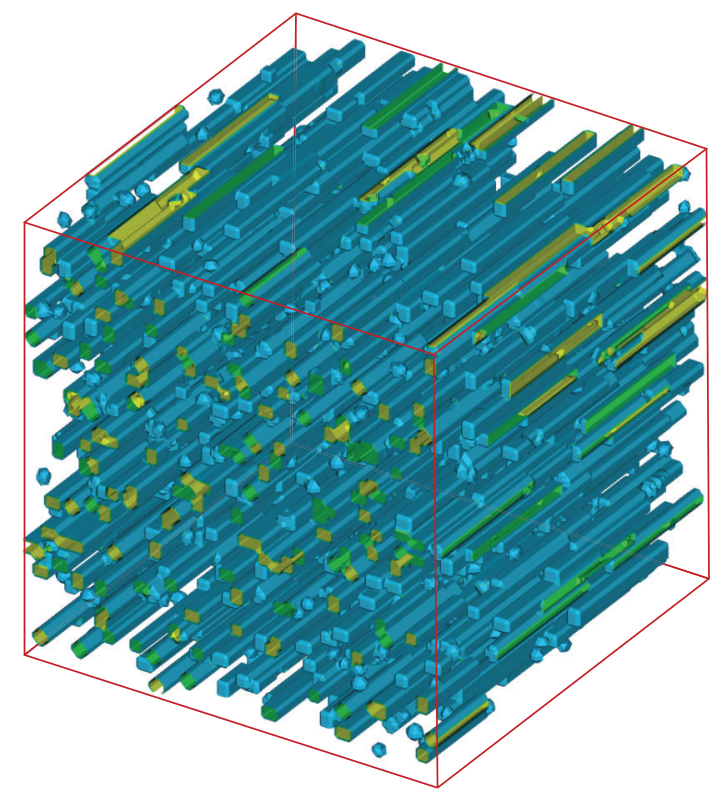

(b)

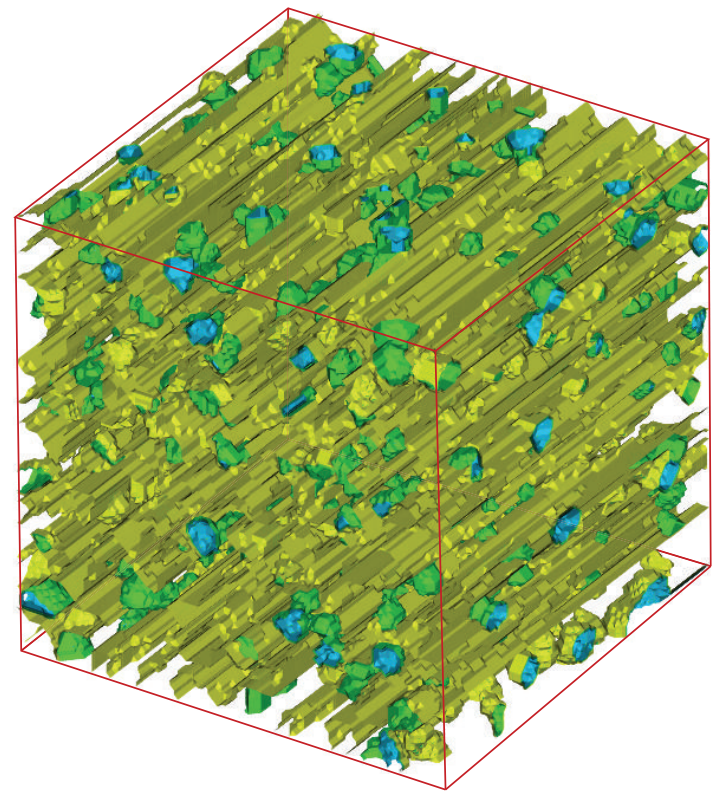

(d)

FIGURE 4: Morphology evolution for each spherulite and shish-kebab.

shish-kebabs. However, its effect to the average length-todiameter ratio of shish-kebab is not significant.

We will also mention that the driving force to the nucleation density of shish-kebabs $N_{s-\mathrm{k}}$ is not well understood. However, it is accepted that it is related to the effect of flow $[1,2]$. Therefore, one can adjust the flow effect to obtain the optimal nucleation density of shish-kebabs.

3.4. Effects of Growth Rate of Spherulites $G_{s}$. In order to study the effects of growth rate of spherulites $G_{s}$ on crystallization kinetics and morphology, three groups of $G_{s}$ are tested; namely, $G_{s}=0.5 \mu \mathrm{m} / \mathrm{s}, 1 \mu \mathrm{m} / \mathrm{s}$, and $2 \mu \mathrm{m} / \mathrm{s}$. We also assume that the radius growth rate of shish-kebabs $G_{s-k, r}$ is equal to the growth rate of spherulites $G_{s}$. The other parameters are fixed in the simulation to allow comparison. Crystallization kinetics data and morphology with different growth rate of spherulites $G_{s}$ are shown in Figure 10. Evidently, an increase in growth rate of spherulites $G_{s}$ speeds up the crystallization rate. Morphology for similar relative crystallinity $(\alpha \approx 0.5)$ with different growth rate of spherulites is also shown in Figure 10. It can be seen that increase the growth rate of spherulites increases the radius of spherulites and shishkebabs. The observed trend is also in the case of final morphology which is not shown here. The effect of growth rate of spherulites in this study (spherulites and shishkebabs coexisting system) is not the same as the system 


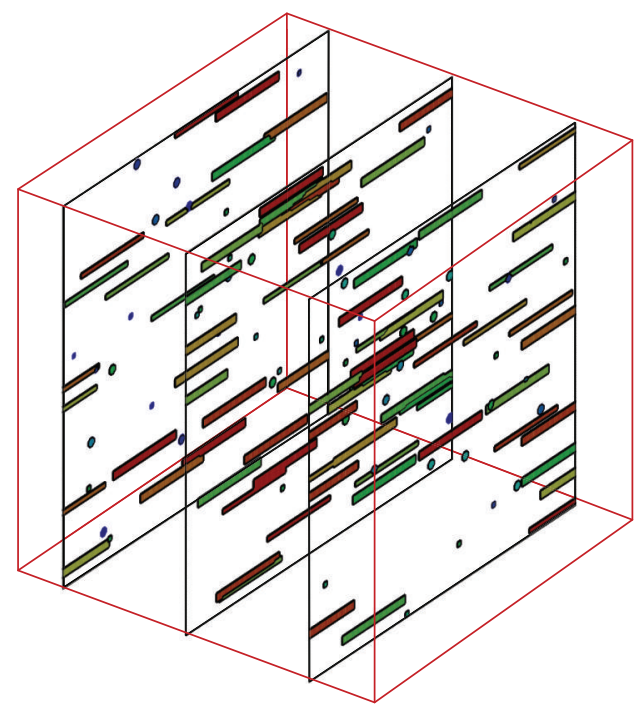

(a)

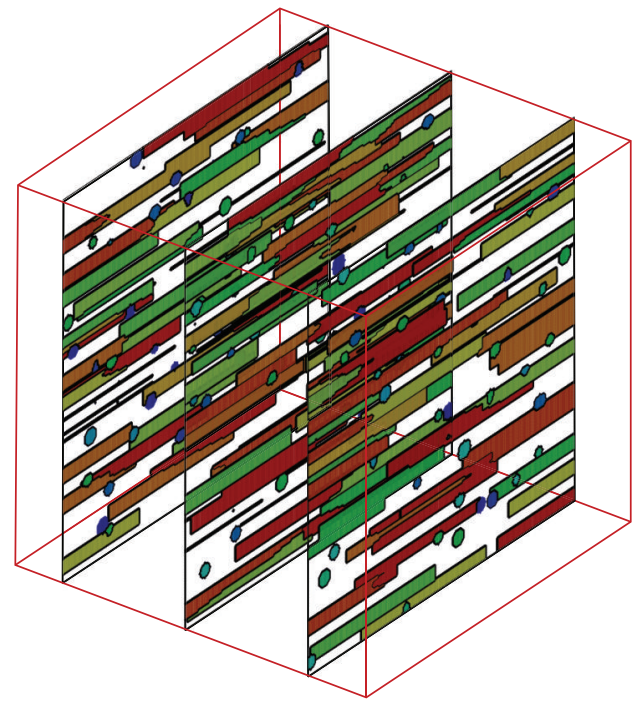

(c)

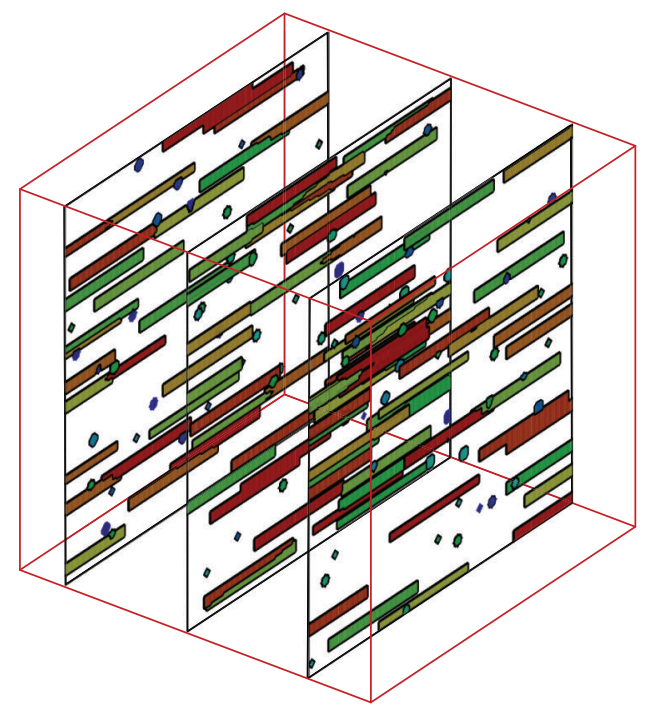

(b)

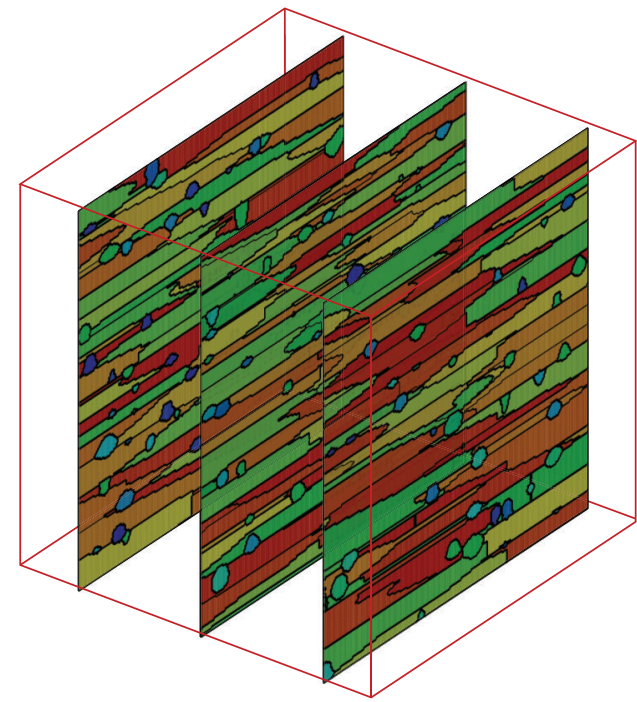

(d)

FIGURE 5: Morphology evolution for spherulites and shish-kebabs at slices.

with only spherulitic structure. When isothermal quiescent crystallization is considered, only spherulites appear and the final morphology is referred to as a Voronoi diagram [11, 13]. It has been reported by many people that Voronoi diagram is only a function of nucleation density of spherulites $N_{s}$ and is independent of growth rate of spherulites $G_{s}$ [11].

In order to know the detailed morphology of crystals, Figure 11 describes the distribution of spherulite size in the final morphology. In the case of higher growth rate of spherulites, the mean radius of spherulites is larger. Table 3 lists the volume fraction and the average length-to-diameter ratio of shish-kebabs. As the growth rate of spherulites increases, the volume fraction of shish-kebabs and the average length-to-diameter ratio of shish-kebabs decrease. Therefore, the shish-kebab is more highly anisotropic with the slower growth rate of spherulites.
The growth rate of spherulites $G_{s}$ is always assumed to be only dependent on temperature since plenty of experiments reported that this parameter is influenced weakly by flow. Therefore, in the real polymer processing, one can change the temperature to adjust the growth rate of spherulites.

3.5. Effects of Length Growth Rate of Shish-Kebabs $G_{\mathrm{s}-\mathrm{k}, l}$. Effects of length growth rate of shish-kebabs $G_{s-k, l}$ on the crystallization kinetics and morphology are also investigated. We test three cases; namely, $G_{\mathrm{s}-\mathrm{k}, l}=5 \mu \mathrm{m} / \mathrm{s}, 10 \mu \mathrm{m} / \mathrm{s}$, and $20 \mu \mathrm{m} / \mathrm{s}$. The corresponding results are plotted in Figure 12. From Figure 12, it is observed that crystallization rate is quicker in the case of higher length growth rate of shishkebabs. Compared to Figure 10, it can be concluded that the effect of length growth rate of shish-kebabs has a weaker impact than the effect of radius growth rate of spherulites 


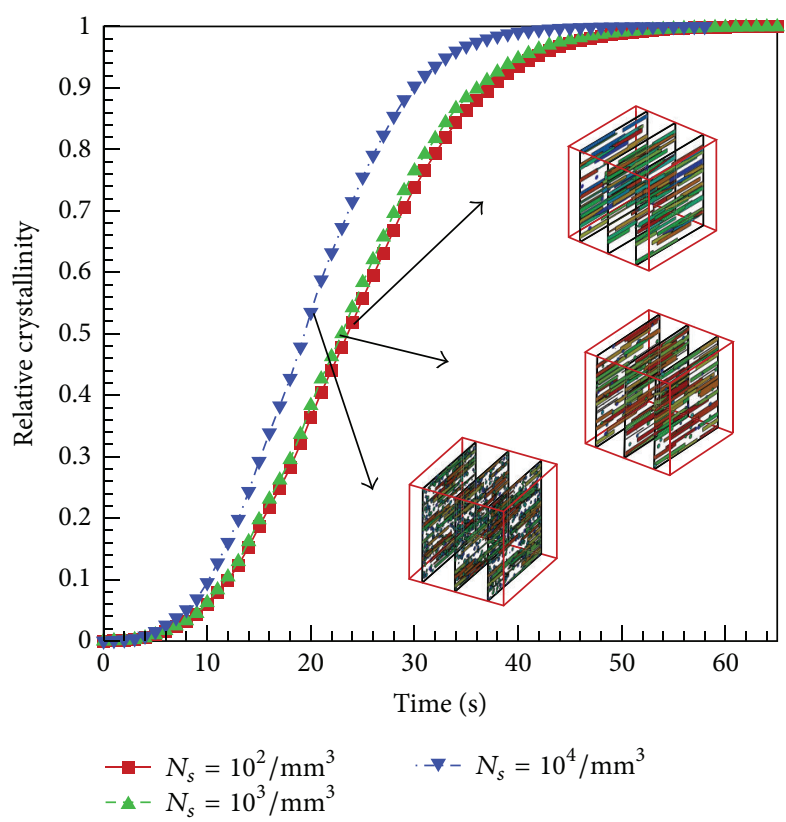

FIGURE 6: Relative crystallinity and morphology comparison with different $N_{s}$.

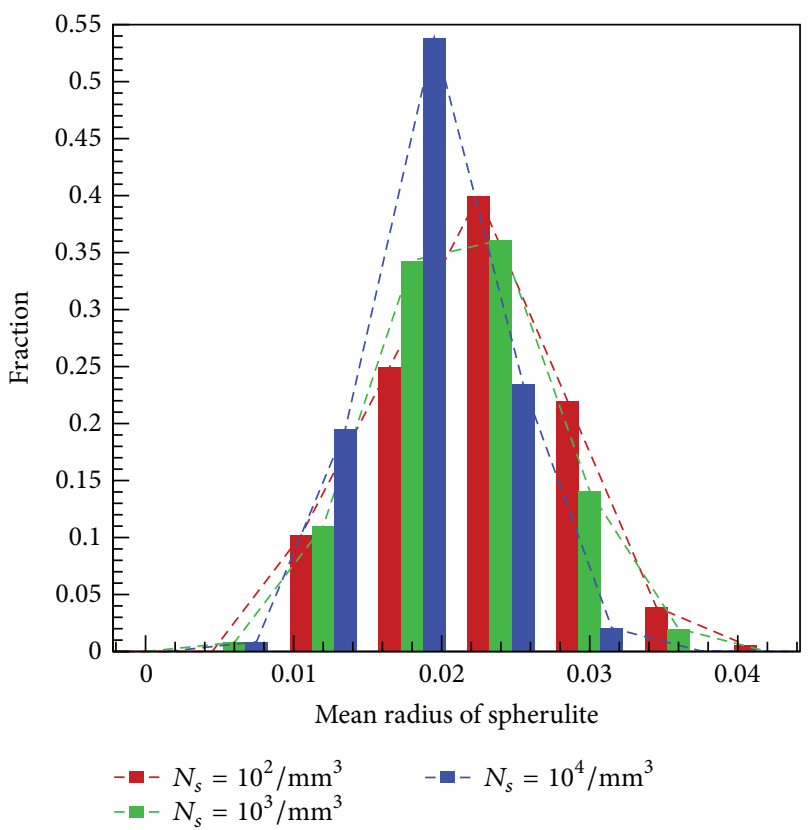

Figure 7: Distribution of spherulite size.

(shish-kebabs) on crystallization rate. This can be derived from the expression of fictive volume of spherulites and shishkebabs by (3) and (4). Since we assume the radius growth rate of shish-kebabs $G_{\mathrm{s}-\mathrm{k}, r}$ to be equal to the radius growth rate of spherulites $G_{s}$, the fictive volume of crystals $\alpha_{f}$ is found to be approximately linear depending on the length growth rate of shish-kebabs $G_{\mathrm{s}-\mathrm{k}, l}$ and to be approximately cubical depending on the radius growth rate of spherulites $G_{s}$. Morphology for similar relative crystallinity $(\alpha \approx 0.5)$ with

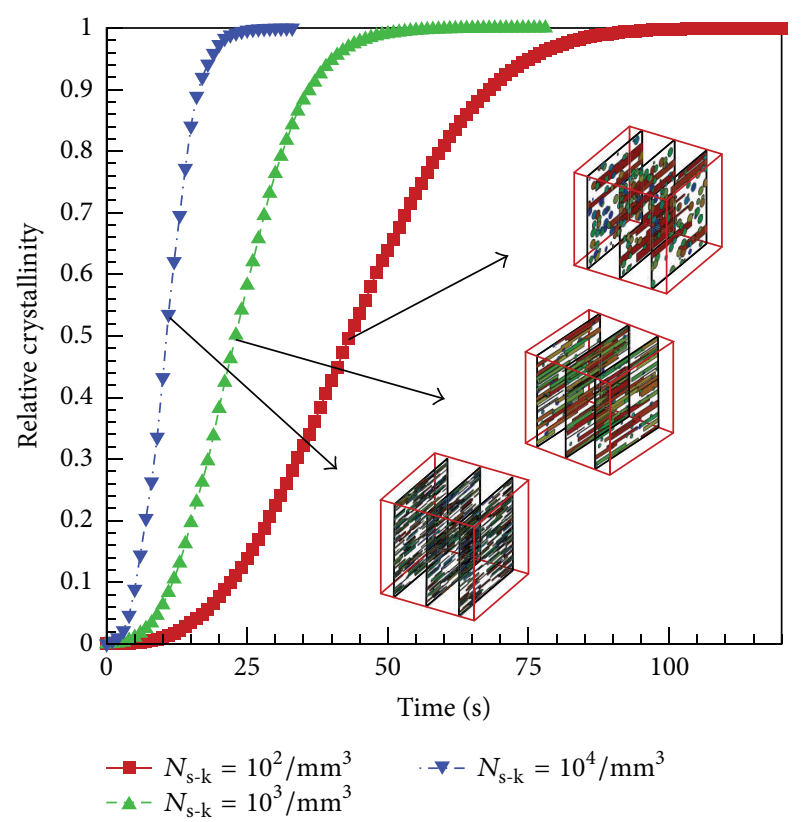

FIGURE 8: Relative crystallinity and morphology comparison with different $N_{\mathrm{s}-\mathrm{k}}$.

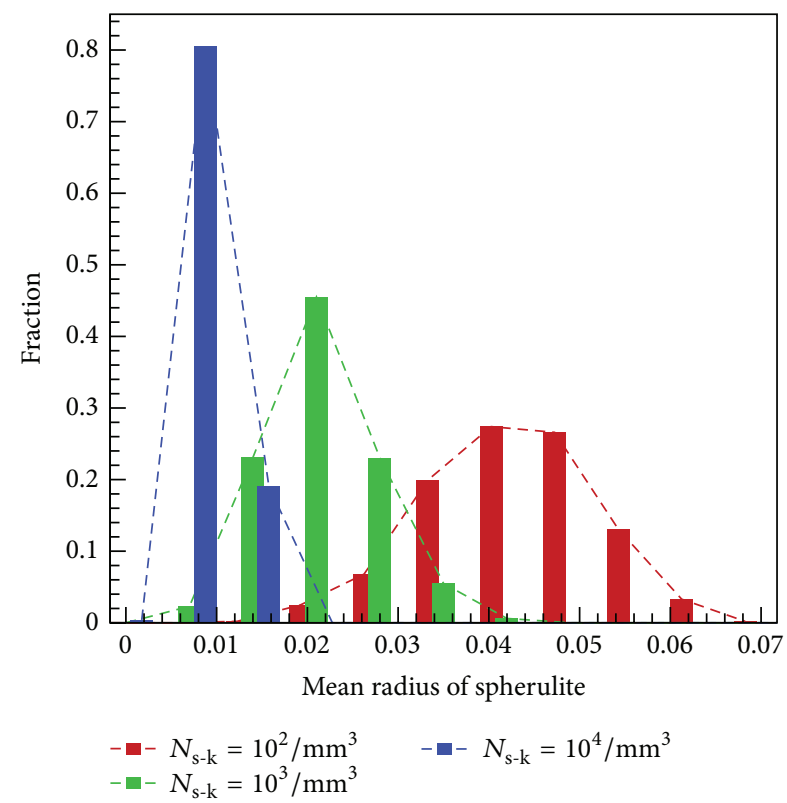

FIGURE 9: Distribution of spherulite size.

different growth rate of length of shish-kebabs is also shown in Figure 12. The observation from Figure 12 tells us that an increase in the length growth rate of shish-kebabs leads to a longer shish-kebab structure in length.

Figure 13 describes the distribution of spherulite size in the final morphology. Mean radius of spherulite size is larger in the case of smaller length growth rate of shish-kebabs. Table 4 lists the volume fraction and the average length-todiameter ratio of shish-kebabs. As the length growth rate of shish-kebabs increases, the volume fraction of shish-kebabs 
TABLE 3: Volume fraction and average length-to-diameter ratio of shish-kebabs.

\begin{tabular}{lccc}
\hline Case & $G_{s}=0.5 \mu \mathrm{m} / \mathrm{s}$ & $G_{s}=1.0 \mu \mathrm{m} / \mathrm{s}$ & $G_{s}=2.0 \mu \mathrm{m} / \mathrm{s}$ \\
\hline Volume fraction of shish-kebabs & $95.4 \%$ & $92.2 \%$ & $85.9 \%$ \\
Average length-to-diameter ratio of shish-kebabs & 13.73 & 7.93 & 4.05 \\
\hline
\end{tabular}

TABLE 4: Volume fraction and average length-to-diameter ratio of shish-kebabs.

\begin{tabular}{lccc}
\hline Case & $G_{\mathrm{s}-\mathrm{k}, l}=5 \mu \mathrm{m} / \mathrm{s}$ & $G_{\mathrm{s}-\mathrm{k}, l}=10 \mu \mathrm{m} / \mathrm{s}$ & $G_{\mathrm{s}-\mathrm{k}, l}=20 \mu \mathrm{m} / \mathrm{s}$ \\
\hline Volume fraction of shish-kebabs & $86.3 \%$ & $92.2 \%$ & $95.2 \%$ \\
Average length-to-diameter ratio of shish-kebabs & 4.21 & 7.93 & 13.2 \\
\hline
\end{tabular}

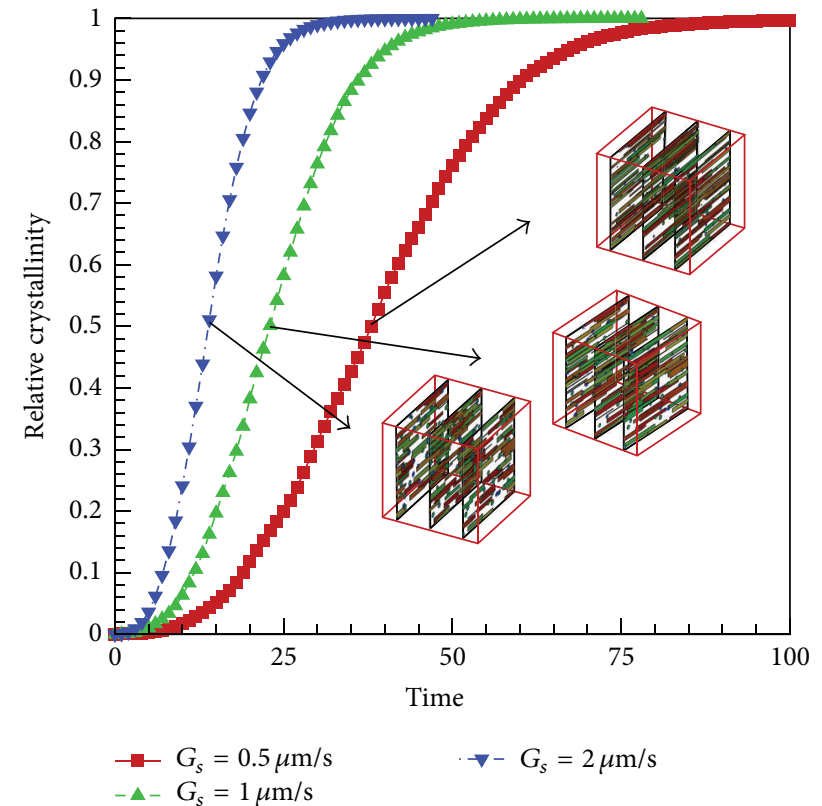

FIGURE 10: Relative crystallinity and morphology comparison with different $G_{s}$.

and the average length-to-diameter ratio of shish-kebabs increase.

Length growth rate of shish-kebabs $G_{s-k, l}$ is always modeled as a function of flow; therefore, in the real polymer processing, one can impose the different flow to change the length growth rate of shish-kebabs.

\section{Conclusions}

A Monte Carlo simulation of morphology and kinetics of spherulites and shish-kebabs in isothermal polymer crystallization is presented here. The simulated crystallization kinetics data show a good agreement with results from the theoretical Avrami model. Thus, the method and simulation results presented here are reliable and valid.

A parametric study is used here to explore the effects of each variable on crystal morphology evolution as well as crystallization kinetics. The effects of nucleation density and growth rate of spherulites, nucleation density, and length growth rate of shish-kebabs, respectively, on crystallization

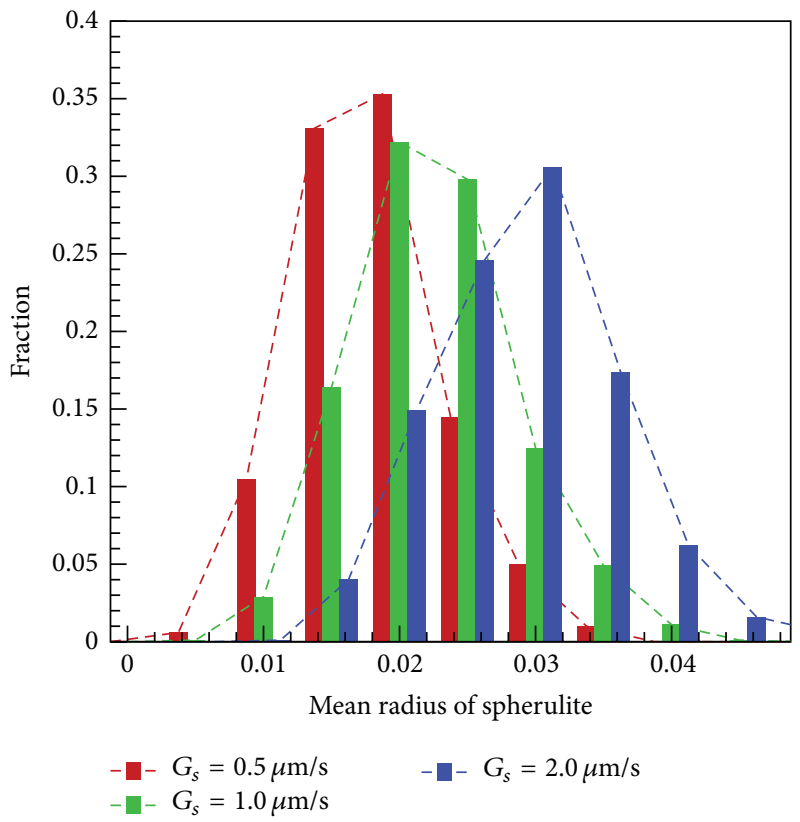

FIGURE 11: Distribution of spherulite size.

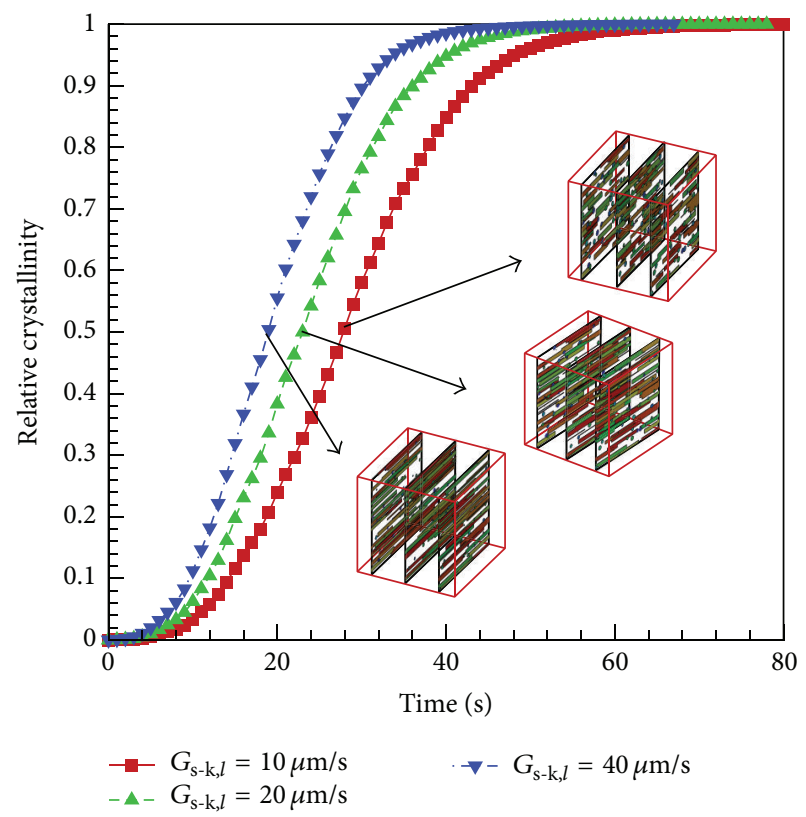

FIGURE 12: Relative crystallinity and morphology comparison with different $G_{s-k, l}$. 


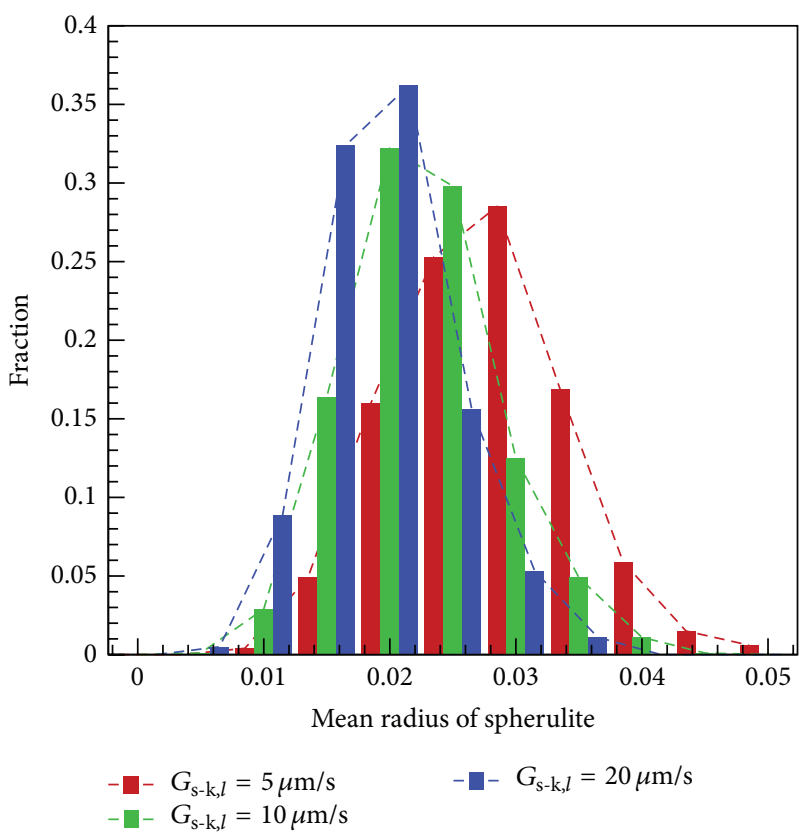

FIGURE 13: Distribution of spherulite size.

are investigated. It has been found that nucleation densities of both spherulites and shish-kebabs have a strong effect on both crystallization rate and morphology. An increase in nucleation density of either spherulites or shish-kebabs leads to a quicker crystallization rate and a smaller average crystal size. It is also shown that nucleation density of shish-kebabs has a stronger impact on crystallization rate. Growth rate of spherulites and length growth rate of shish-kebabs also significantly affect the crystallization rate and morphology. An increase in growth rate of spherulites or length growth rate of shish-kebabs also speeds up the crystallization rate; additionally, a decrease in growth rate of spherulites or an increase in length growth rate of shish-kebabs results in a more highly anisotropic shish-kebab structure and a smaller average spherulite structure. Results also show that the effect of growth rate of spherulites is more important than the effect of length growth rate of shish-kebabs on crystallization.

We present a computer simulation for isothermal crystallization of spherulites and shish-kebabs in polymer system. The computational approach presented here allows us to separately examine the effect of each parameter which cannot be done in experiments as several parameters change simultaneously. Therefore, results presented here may provide some new insights on processing improvement. Our future work will be concentrated on the morphology development and kinetics prediction in polymer manufacture processing which takes into account the temperature and flow.

\section{Conflict of Interests}

The authors declare that there is no conflict of interests regarding the publication of this paper.

\section{Acknowledgments}

The financial supports provided by the Natural Sciences Foundation of China (no. 11402078), Postdoctoral Science Foundation of China (no. 2012M521403), and the Scientific and Technological Research Project of Henan Province (nos. 122102210198, 14B110020) are fully acknowledged.

\section{References}

[1] H. Zuidema, Flow induced crystallization of polymers: application to injection moulding [Ph.D. thesis], Eindhoven University of Technology, Eindhoven, The Netherlands, 2000.

[2] R. Pantani, I. Coccorullo, V. Speranza, and G. Titomanlio, "Modeling of morphology evolution in the injection molding process of thermoplastic polymers," Progress in Polymer Science, vol. 30, no. 12, pp. 1185-1222, 2005.

[3] A. K. Doufas, A. J. McHugh, and C. Miller, "Simulation of melt spinning including flow-induced crystallization. Part I. Model development and predictions," Journal of Non-Newtonian Fluid Mechanics, vol. 92, no. 1, pp. 27-66, 2000.

[4] R. I. Tanner and F. Z. Qi, "A comparison of some models for describing polymer crystallization at low deformation rates," Journal of Non-Newtonian Fluid Mechanics, vol. 127, no. 2-3, pp. 131-141, 2005.

[5] A. Ziabicki, L. Janecki, and A. Sorrentino, "The role of flowinduced crystallisation in melt spinning," e-Ploymers, vol. 4, no. 1, pp. 823-836, 2004.

[6] H. Zuidema, G. W. M. Peters, and H. E. H. Meijer, "Development and validation of a recoverable strain-based model for flow-induced crystallization of polymers," Macromolecular Theory and Simulations, vol. 10, no. 5, pp. 447-460, 2001.

[7] E. Koscher and R. Fulchiron, "Influence of shear on polypropylene crystallization: morphology development and kinetics," Polymer, vol. 43, no. 25, pp. 6931-6942, 2002.

[8] R. Zheng and P. K. Kennedy, "A model for post-flow induced crystallization: general equations and predictions," Journal of Rheology, vol. 48, no. 4, pp. 823-842, 2004.

[9] S. Swaminarayan and C. Charbon, "A multiscale model for polymer crystallization. I: growth of individual spherulites," Polymer Engineering and Science, vol. 38, no. 4, pp. 634-643, 1998.

[10] V. Capasso, Mathematical Modelling for Polymer Processing, Springer, Berlin, Germany, 2003.

[11] A. Micheletti and M. Burger, "Stochastic and deterministic simulation of nonisothermal crystallization of polymers," Journal of Mathematical Chemistry, vol. 30, no. 2, pp. 169-193, 2001.

[12] S. Ketdee and S. Anantawaraskul, "Simulation of crystallization kinetics and morphological development during isothermal crystallization of polymers: effect of number of nuclei and growth rate," Chemical Engineering Communications, vol. 195, no. 11, pp. 1315-1327, 2008.

[13] D. Raabe, "Mesoscale simulation of spherulite growth during polymer crystallization by use of a cellular automaton," Acta Materialia, vol. 52, no. 9, pp. 2653-2664, 2004.

[14] D. Raabe and A. Godara, "Mesoscale simulation of the kinetics and topology of spherulite growth during crystallization of isotactic polypropylene (iPP) by using a cellular automaton," Modelling and Simulation in Materials Science and Engineering, vol. 13, no. 5, pp. 733-751, 2005. 
[15] J. X. Lin, C. Y. Wang, and Y. Y. Zheng, "Prediction of isothermal crystallization parameters in monomer cast nylon 6," Computers and Chemical Engineering, vol. 32, no. 12, pp. 3023-3029, 2008.

[16] C. Ruan, "Multiscale numerical study of 3D polymer crystallization during cooling stage," Mathematical Problems in Engineering, vol. 2012, Article ID 802420, 15 pages, 2012.

[17] C. Ruan, J. Ouyang, and S. Liu, "Multi-scale modeling and simulation of crystallization during cooling in short fiber reinforced composites," International Journal of Heat and Mass Transfer, vol. 55, no. 7-8, pp. 1911-1921, 2012.

[18] C. Ruan, J. Ouyang, S. Liu, and L. Zhang, "Computer modeling of isothermal crystallization in short fiber reinforced composites," Computers and Chemical Engineering, vol. 35, no. 11, pp. 2306-2317, 2011.

[19] M. Avrami, "Kinetics of phase change. II. Transformation-time relations for random distribution of nuclei," The Journal of Chemical Physics, vol. 8, no. 2, pp. 212-224, 1940.

[20] M. Avrami, "Granulation, phase change, and microstructure kinetics of phase change. III," The Journal of Chemical Physics, vol. 9, no. 2, pp. 177-184, 1941.

[21] M. Avrami, "Kinetics of phase change. I. General theory," The Journal of Chemical Physics, vol. 7, no. 12, pp. 1103-1112, 1939.

[22] G.-Q. Zheng, W. Yang, M.-B. Yang, J.-B. Chen, Q. Li, and C.-Y. Shen, "Gas-assisted injection molded polypropylene: the skincore structure," Polymer Engineering and Science, vol. 48, no. 5, pp. 976-986, 2008.

[23] Y.-G. Zhou, L.-S. Turng, and C.-Y. Shen, "Modeling and prediction of morphology and crystallinity for cylindrical-shaped crystals during polymer processing," Polymer Engineering \& Science, vol. 50, no. 6, pp. 1226-1235, 2010. 


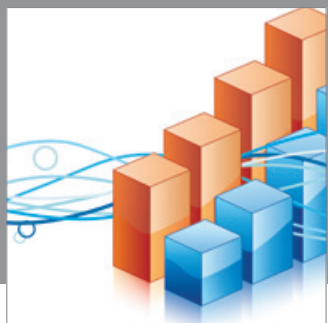

Advances in

Operations Research

mansans

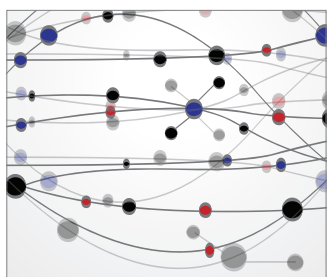

The Scientific World Journal
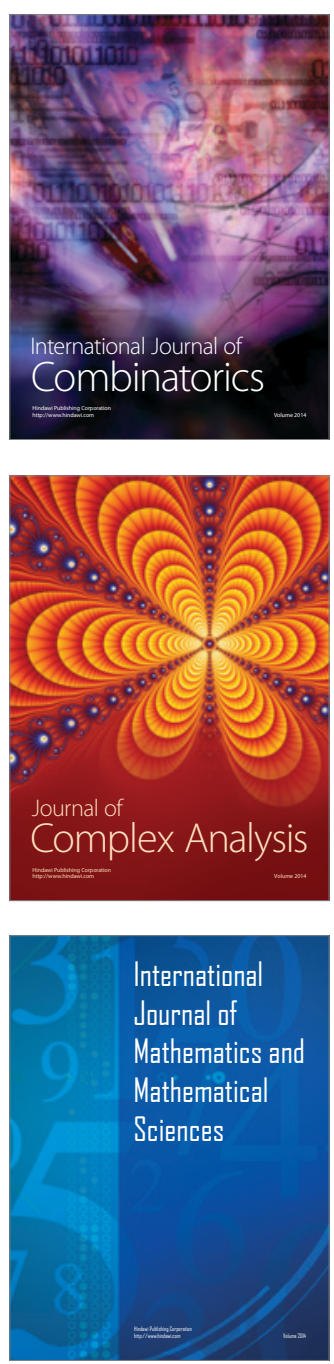
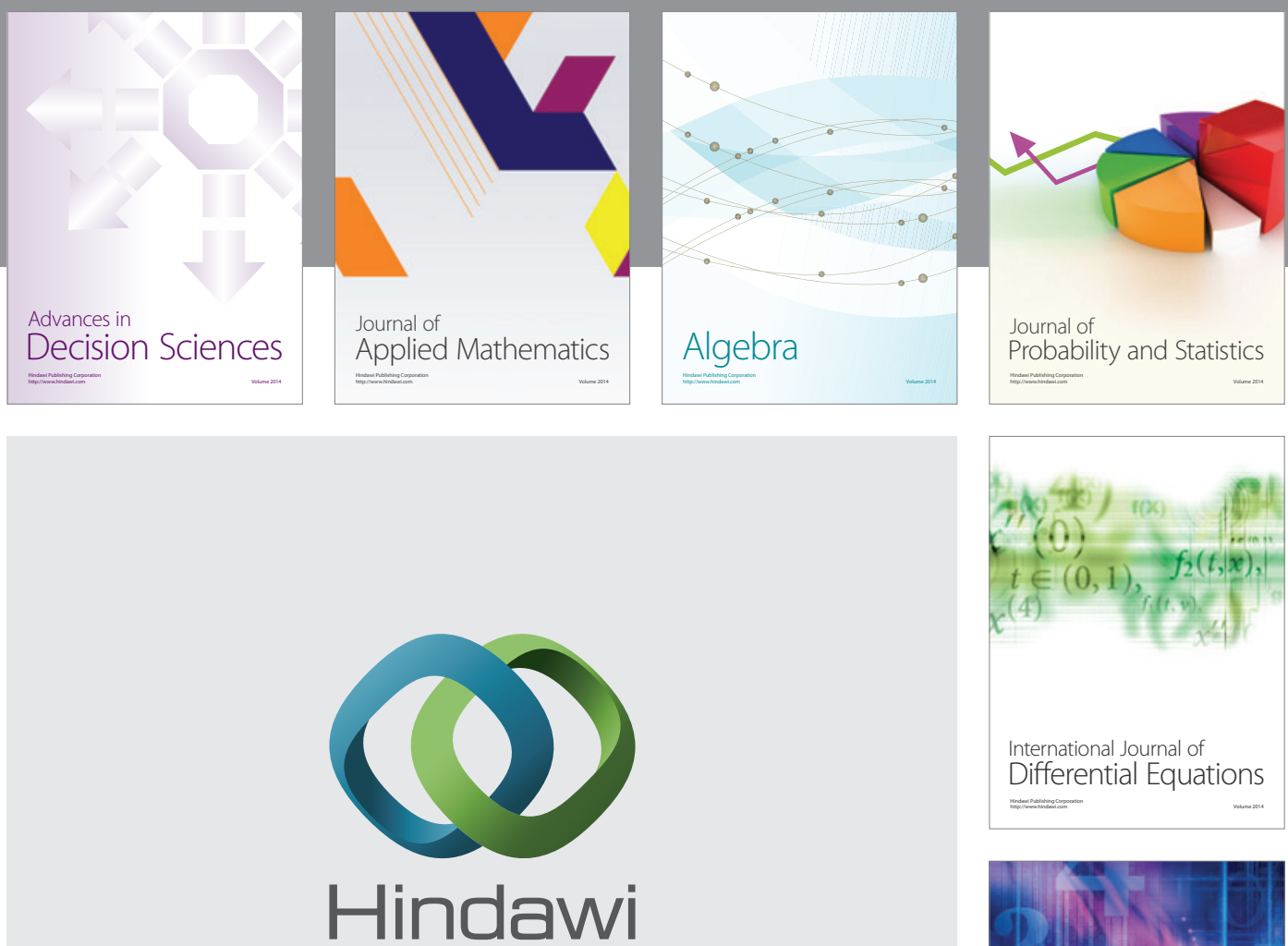

Submit your manuscripts at http://www.hindawi.com
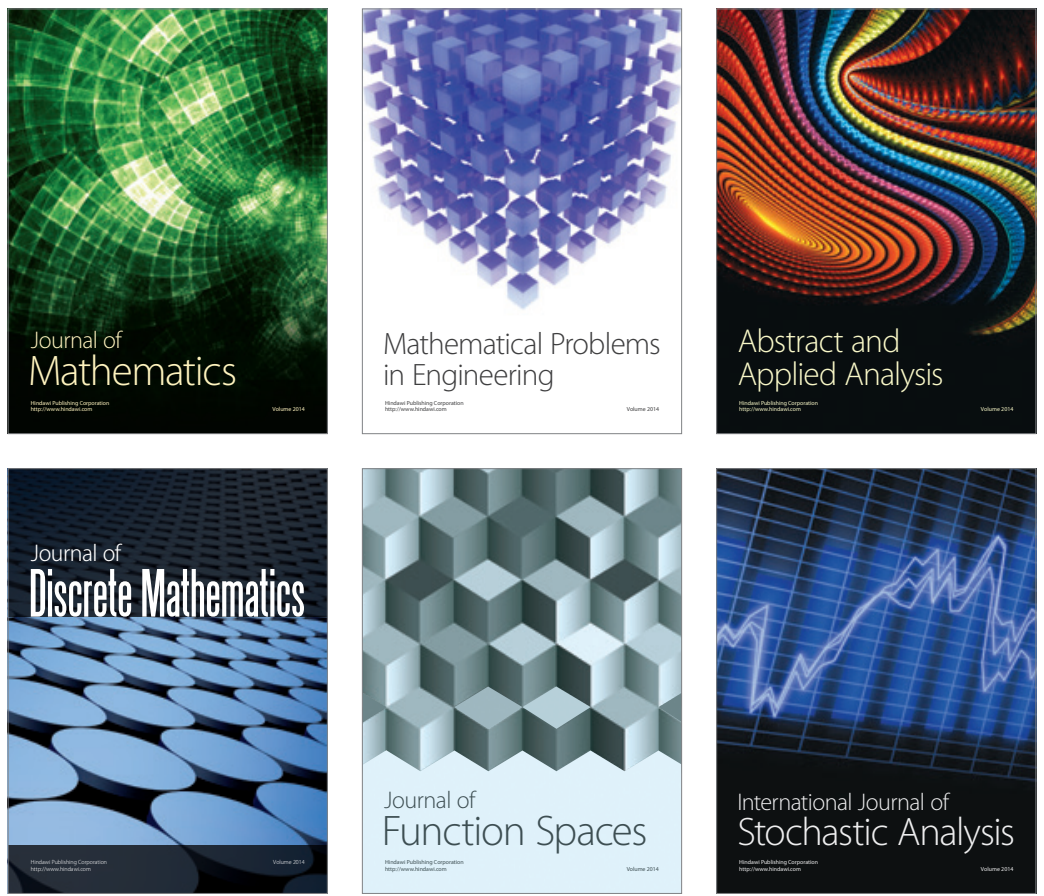

Journal of

Function Spaces

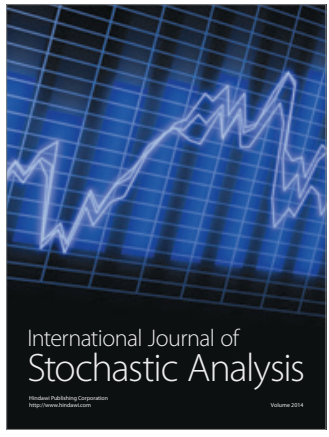

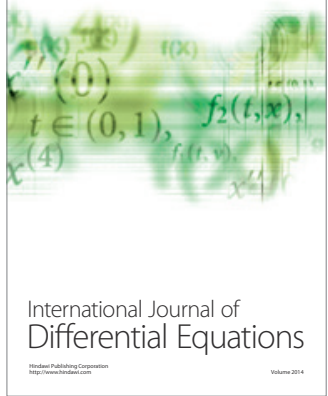
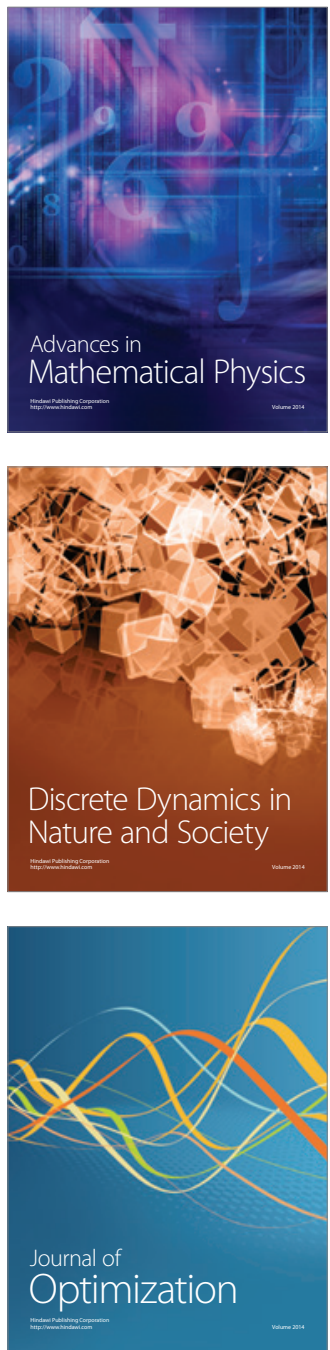